\title{
Correction to: Parenting style as an investment in human development
}

\section{Deborah A. Cobb-Clark ${ }^{1,2,3}$ • Nicolás Salamanca ${ }^{2,3,4} \cdot$ Anna Zhu ${ }^{2,3,4}$}

Published online: 11 April 2020

(C) Springer-Verlag GmbH Germany, part of Springer Nature 2020

Correction to: J Popul Econ (2019) 32:1315-1352.

\section{https://doi.org/10.1007/s00148-018-0703-2}

It has been brought to our attention that the comparative statics derived in Section 3.3.1 of our paper and presented in Table 1 (p. 1326) are wrong.

To see this, it is easiest to first re-express eqs. (11a) through (11c) as:

$$
\begin{gathered}
\mathrm{R}_{\mathrm{x}}^{\mathrm{t}}=\frac{\frac{\partial \mathrm{Q}}{\frac{\partial \mathrm{Z}_{\mathrm{t}}}{\partial \mathrm{Q}}}=\frac{\mathrm{w}}{\mathrm{p} \mathrm{Z}_{\mathrm{x}}} \cdot\left(\mathrm{A}^{\mathrm{P}}-\mathrm{a}\right) \cdot\left(\frac{\frac{\mathrm{dt}}{\mathrm{d} \mathrm{Z}_{\mathrm{t}}}}{\frac{\mathrm{dx}}{\mathrm{d} \mathrm{Z}_{\mathrm{x}}}}\right)=\frac{\mathrm{w}}{\mathrm{p}} \cdot \mathrm{a}_{\mathrm{w}} \cdot \psi_{\mathrm{x}}^{\mathrm{t}}}{\mathrm{R}_{\mathrm{x}}^{\mathrm{a}}=\frac{\frac{\partial \mathrm{Q}}{\partial \mathrm{Z}_{\mathrm{a}}}}{\frac{\partial \mathrm{Q}}{\partial \mathrm{Z}_{\mathrm{x}}}}=\frac{\mathrm{w}}{\mathrm{p}} \cdot\left(\mathrm{T}^{\mathrm{P}}-\mathrm{t}\right) \cdot\left(\frac{\frac{\mathrm{da}}{\mathrm{d} \mathrm{Z}_{\mathrm{a}}}}{\frac{\mathrm{dx}}{\mathrm{d} \mathrm{Z}_{\mathrm{x}}}}\right)=\frac{\mathrm{w}}{\mathrm{p}} \cdot \mathrm{t}_{\mathrm{w}} \cdot \psi_{\mathrm{x}}^{\mathrm{a}}}
\end{gathered}
$$

The online version of the original article can be found at https://doi.org/10.1007/s00148-018-0703-2

Nicolás Salamanca

n.salamanca@unimelb.edu.au

1 School of Economics, The University of Sydney, Sydney, Australia

2 ARC Centre of Excellence for Children and Families over the Life Course, Brisbane, Australia

3 Institute of Labor Economics (IZA), Bonn, Germany

4 Melbourne Institute of Applied Economic and Social Research, The University of Melbourne, Melbourne, Australia 


$$
\mathrm{R}_{\mathrm{t}}^{\mathrm{a}}=\frac{\frac{\partial \mathrm{Q}}{\frac{\partial \mathrm{Z}_{\mathrm{a}}}{\partial \mathrm{Q}}}}{\frac{\partial \mathrm{Z}_{\mathrm{t}}}{\mathrm{w}} \cdot\left(\mathrm{A}^{\mathrm{P}}-\mathrm{a}\right)}\left(\frac{\mathrm{w} \cdot\left(\mathrm{T}^{\mathrm{P}}-\mathrm{t}\right)}{\frac{\mathrm{da}}{\mathrm{dt}}}\right)=\frac{\mathrm{t}_{\mathrm{w}}}{\mathrm{a}_{\mathrm{w}}} \cdot \psi_{\mathrm{t}}^{\mathrm{a}}
$$

From these equations, it is more straightforward to arrive at the corrected Table 1:

Corrected Table 1.

Comparative statics of parenting style and traditional models.

\begin{tabular}{|c|c|c|c|c|}
\hline \multirow{4}{*}{$\begin{array}{l}\text { Partial derivative of: } \\
R_{x}^{t}\end{array}$} & \multirow{4}{*}{$\begin{array}{l}\text { In model: } \\
\text { Parenting } \\
\text { Traditional }\end{array}$} & \multicolumn{3}{|l|}{ With respect to: } \\
\hline & & $\frac{\partial}{\partial A^{P}}$ & $\frac{\partial}{\partial A^{P}}$ & $\frac{\partial}{\partial A^{P}}$ \\
\hline & & $\frac{\mathrm{w}}{\mathrm{w}} \psi_{\mathrm{x}}^{\mathrm{t}} \frac{\partial \mathrm{a}_{\mathrm{w}}}{\partial \mathrm{A}^{\mathrm{P}}}$ & $\frac{\mathrm{w}}{\mathrm{p}^{2}} \psi_{\mathrm{x}}^{\mathrm{t}}\left(\frac{\partial \mathrm{a}_{\mathrm{w}}}{\partial \mathrm{p}} \mathrm{p}-\mathrm{a}_{\mathrm{w}}\right)$ & $\frac{\psi_{x}^{t}}{p}\left(a_{w}+w \frac{\partial a_{w}}{\partial w}\right)$ \\
\hline & & 0 & $-\left(\frac{\mathrm{w}}{\mathrm{p}^{2}} \psi_{\mathrm{x}}^{\mathrm{t}}\right)$ & $\left(\frac{\psi_{x}^{t}}{p}\right)$ \\
\hline \multirow[t]{2}{*}{$R_{x}^{a}$} & Parenting & $\frac{\mathrm{w}}{\mathrm{p}} \psi_{\mathrm{x}}^{\mathrm{a}} \frac{\partial \mathrm{t}_{\mathrm{w}}}{\partial \mathrm{A}^{\mathrm{P}}}$ & $\frac{\mathrm{w}}{\mathrm{p}^{2}} \psi_{\mathrm{x}}^{\mathrm{a}}\left(\frac{\partial \mathrm{t}_{\mathrm{w}}}{\partial \mathrm{p}} \mathrm{p}-\mathrm{t}_{\mathrm{w}}\right)$ & $\frac{\psi_{x}^{\mathrm{a}}}{\mathrm{p}}\left(\mathrm{t}_{\mathrm{w}}+\mathrm{w} \frac{\partial \mathrm{t}_{\mathrm{w}}}{\partial \mathrm{w}}\right)$ \\
\hline & Traditional & N/A & $\mathrm{N} / \mathrm{A}$ & N/A \\
\hline \multirow[t]{2}{*}{$R_{t}^{a}$} & Parenting & $\frac{\psi_{t}^{a}}{a_{w}^{2}}\left(\frac{\partial \mathrm{t}_{\mathrm{w}}}{\partial \mathrm{A}^{\mathrm{P}}} \mathrm{w}_{\mathrm{w}}-\mathrm{t}_{\mathrm{w}} \frac{\partial \mathrm{a}_{\mathrm{w}}}{\partial \mathrm{A}^{\mathrm{p}}}\right)$ & $\frac{\psi_{t}^{a}}{a_{w}^{2}}\left(\frac{\partial t_{\mathrm{w}}}{\partial \mathrm{p}} \mathrm{a}_{\mathrm{w}}-\mathrm{t}_{\mathrm{w}} \frac{\partial \mathrm{a}_{\mathrm{w}}}{\partial \mathrm{p}}\right)$ & $\frac{\psi_{t}^{a}}{a_{w}^{2}}\left(\frac{\partial t_{w}}{\partial w} a_{w}-t_{w} \frac{\partial a_{w}}{\partial w}\right)$ \\
\hline & Traditional & N/A & N/A & N/A \\
\hline
\end{tabular}

The discussion of the comparative statistics (p. 1326) remains unaffected by this correction. A corrected Appendix for the paper can be found here.

* We thank Linfeng Fan at the School of Labor and Human Recourses of Renming University of China for carefully going through our derivations and making us aware of this mistake, and Tiffany Ho for verifying our corrections in this errata. 